\title{
Análise de amortecimento em decremento logarítmico com acelerômetro de vibração
}

\author{
Damping analysis in logarithmic decrement with vibration accelerometer \\ Análisis de amortiguación en decremento logarítmico con acelerómetro de vibraciones
}

Recebido: 14/08/2021 | Revisado: 20/08/2021 | Aceito: 28/08/2021 | Publicado: 29/08/2021

David Augusto Ribeiro

ORCID: https://orcid.org/0000-0002-2888-2928 Universidade Federal de Lavras, Brasil

E-mail: david.augusto.ribeiro@gmail.com

Márcio Wladimir Santana

ORCID: https://orcid.org/0000-0002-7673-3724 Universidade Federal de Lavras, Brasil

E-mail: marciosantana@cefetmg.br

Dennis Santos Tavares

ORCID: https://orcid.org/0000-0001-5990-9516 Universidade Federal de Lavras, Brasil

E-mail: dennis_santostavares@yahoo.com.br

Pablo Henrique Machado de Souza

ORCID: https://orcid.org/0000-0003-1196-4676 Universidade Federal de Lavras, Brasil E-mail: pablo.souza@estudante.ufla.br

Kissia Juamara Pereira Marques

ORCID: https://orcid.org/0000-0001-8461-5114 Universidade Federal de Lavras, Brasil E-mail: kissiastma@gmail.com

Lohayne Cristina de Abreu

ORCID: https://orcid.org/0000-0002-3778-2531 Universidade Federal de Lavras, Brasil E-mail: lohaynecristina@yahoo.com.br Ana Carolina Corrêa Furtini

ORCID: https://orcid.org/0000-0002-2106-6602 Universidade Federal de Lavras, Brasil

E-mail: carol.furtini@gmail.com

Renata Lopes Rosa

ORCID: https://orcid.org/0000-0002-7595-7187 Universidade Federal de Lavras, Brasil

E-mail: renata.rosa@ufla.br

Demóstenes Zegarra Rodríguez

ORCID: https://orcid.org/0000-0001-5401-7551 Universidade Federal de Lavras, Brasil E-mail: demostenes.zegarra@ufla.br

\begin{abstract}
Resumo
O amortecimento é uma propriedade dos materiais que pode ocorrer em aspecto de escala micro e macroscópica. Há um crescimento no conceito da análise de marcadores de trincas internas estruturais que causam danos em materiais diversos, devido sua ampla aplicabilidade na resolução de problemas. No processo de amortecimento ocorre a perda de energia do material quando este entra em contato físico com outro material, no qual o fenômeno pode ser observado por meio de equipamentos modernos de alta sensibilidade sob ambiente controlado. Há poucos institutos de pesquisa com capacidade de análises em ensaios de amortecimento precisos com corpos de prova. O uso de dispositivo acelerômetro contorna a problemática do alto custo laboratorial envolvidos e permite maior acessíbilidade. Objetiva-se apresentar uma metodologia de análise de parâmetros associados ao fenômeno de amortecimento, fazendo-se uso de algotítmos para acesso aberto e que permita sua replicação em modelos físicos. O trabalho apresentou efciência em determinar a frequência e parâmetros associados de sinais de corpos de prova.
\end{abstract}

Palavras-chave: Amortecimento; Materiais; Acelerômetro; Metodologia; Algoritmos.

\section{Abstract}

Damping is a property of materials that can occur on both a micro and macroscopic scale. There is a growth in the concept of analysis of structural internal crack markers that cause damage in different materials, due to its wide applicability in problem solving. In the damping process, the material loses energy when it comes into physical contact with another material, in which the phenomenon can be observed by means of modern equipment with high sensitivity under a controlled environment. There are few research institutes capable of analyzing accurate damping tests with 
specimens. The use of an accelerometer device circumvents the problem of the high laboratory cost involved and allows for greater accessibility. The objective is to present a methodology for analyzing parameters associated with the damping phenomenon, making use of algorithms for open access and allowing its replication in physical models. The work was efficient in determining the frequency and associated parameters of specimen signals.

Keywords: Damping; Materials; Accelerometer; Methodology; Algorithms.

\section{Resumen}

La amortiguación es una propiedad de los materiales que puede ocurrir tanto a escala micro como macroscópica. Existe un crecimiento en el concepto de análisis de marcadores de fisuras internas estructurales que causan daños en diferentes materiales, debido a su amplia aplicabilidad en la resolución de problemas. En el proceso de amortiguación, el material pierde energía cuando entra en contacto físico con otro material, en el que el fenómeno se puede observar mediante equipos modernos de alta sensibilidad en un ambiente controlado. Hay pocos institutos de investigación capaces de analizar pruebas de amortiguación precisas con muestras. El uso de un dispositivo acelerómetro evita el problema del alto costo de laboratorio involucrado y permite una mayor accesibilidad. El objetivo es presentar una metodología para analizar parámetros asociados al fenómeno de amortiguamiento, haciendo uso de algoritmos de acceso abierto y permitiendo su replicación en modelos físicos. El trabajo fue eficiente para determinar la frecuencia y los parámetros asociados de las señales de las muestras.

Palabras clave: Mojadura; Materiales; Acelerómetro; Metodología; Algoritmos.

\section{Introdução}

O amortecimento é uma propriedade dos materiais. Pode ocorrer tanto em aspecto de escala micro ou macro. No processo de amortecimento ocorre a perda de energia do material quando este entra em contato com outro material. Essa propriedade define a amplitude e tempo de vibração do material sob uma excitação. Seus estudos são amplamente encontrados na área civil e material, onde utiliza-se os conceitos em ensaios laboratoriais para posterior aplicação. Por ser tratar de um fenômeno que gera e propaga micro trincas internas, é também muito empregado na análise de resistência material.

De acordo com Singiresu (2009), em muitos sistemas práticos a energia de vibração é gradativamente convertida em calor ou som. Em virtude da redução da energia, a resposta, tal como o deslocamento do sistema, diminui gradativamente. O mecanismo pelo qual a energia de vibração é gradativamente convertida em calor ou som é conhecido como amortecimento. Embora a quantidade de energia convertida em calor ou som seja relativamente pequena, é importante considerar o amortecimento para uma previsão precisa da resposta de vibração de um sistema. Admite-se que um amortecedor não tem nem massa nem elasticidade, e que a força de amortecimento só existe se houver uma velocidade relativa entre as suas duas extremidades. É difícil determinar as causas do amortecimento em sistemas práticos. Como consequência, ele é modelado como um ou mais dos tipos: viscoso, por atrito seco ou sólido.

Para Lazan (1968), os fundamentos se baseiam na existência de pelo menos uma força não conservativa no sistema mecânico, realizando trabalho, implica uma perda de energia mecânica, portanto a ocorrência de amortecimento. O autor define como sendo uma das propriedades mais sensíveis de materiais e estruturas. Existem diversos métodos para determinação do amortecimento, os quais podem ser obtidos basicamente por dois caminhos: mediante a duração da resposta do sistema a uma excitação transitória (exemplo: método do decremento logarítmico) e em função da resposta do sistema em função da frequência (exemplo: método da largura de meia banda de potência). O método do decremento logarítmico calcula o amortecimento a partir da atenuação da resposta acústica do material ou estrutura após uma excitação por impulso. O método da largura de meia banda de potência calcula o amortecimento através da análise da frequência do sinal oriundo da vibração, a partir da relação entre a largura de banda e a frequência central de uma ressonância. Ambos os métodos consideram um modelo para os cálculos, normalmente o modelo de amortecimento viscoelástico. A escolha do método depende principalmente da faixa do amortecimento e da frequência de vibração (Cossolino \& Pereira, 2010).

Aplicações como a de Lima, Lopes e Filho (2021), que utilizaram dispositivos inerciais (acelerômetros) de baixo custo em trechos ferroviários de forma a monitorar as condições dos sistemas, e otimizar sua manutenção, são interessantes métodos. Dispositivos e sensores diversos podem ser utilizados na avaliação econômica de modelos e sistemas logísticos, e aplicá-los para 
avaliações volumes diários de veículos (Valinho et al., 2020). O uso de sensores de vibração ou acelerômetros tem crescido exponencialmente nos últimos anos, e suas aplicações são amplas e cada vez mais necessárias, tais como em análises de vazamentos de água em dutos de escoamento (Ismail et al., 2019), em transformadores de distribuição de energia de alta tensão (Anwar et al., 2018), análise e medição de grandes vibrações em máquinas rotativas tais como rotores como nos estudos de Koene, Viitala e Kuosmanen (2019).

Outro fator importante são as tecnologias para suporte aos acelerômetros, no qual podemos destacar filtros para otimização de vibração para modelos voltados a MEMS (Sistema-Micro-Eletro-Mecânicos) que se baseiam na diminuição de ruídos (Hoang \& Pietrosanto, 2021). No setor de engenharia civil podemos destacar o uso dos sensores, uma vez que uma das principais causas de danos estruturais são as vibrações. Portanto podemos usar os dispositivos para medir o quanto o fenômeno afeta precocemente as estruturas (Hasibuzzaman et al., 2020). Aplicações para análise de inspeção em estruturas de vigas de aço envelhecidas podem ser observadas nos trabalhos de Nakamura, Higashi, Masuda e Tanaka (2018). Quando tratamos desses sensores lidamos com modelos de eixo único ou de três eixos. Nos modelos mais robustos trabalha-se com aceleração convertida do espectro pela transformada de Fourier, obtendo-se o espectro de deslocamento (Liu, Wang \& Tian, 2017).

Mohanty et al. (2014) abordaram o desafio da seleção do tipo específico dos sensores disponíveis no mercado para cada tipo de aplicação específica na indústria, especialmente em tornos mecânicos, onde se analisa espectros em diferentes velocidades de operação. A área de controle e automação também faz uso sensores de vibração, tais como sistemas de controle de azimute em antenas e sistemas de posicionamento de telescópios em realimentação de malha fechada para reconstrução de posição (Keck, Pott \& Sawodny, 2015). Um novidade dos últimos anos são aplicações em CIs (Circuitos Integrados) como o trabalho de Thukral et al. (2019), que utilizaram sensores acelerômetros na medição de vibração em circuitos como PCBs no intuito de obter avaliação da confiabilidade das juntas de soldas dos componentes sujeitos à ruptura por determinadas cargas de vibração.

Em uma análise ampla consideramos parâmetros associados tais como: período do sinal $\left(\mathrm{T}_{\mathrm{d}}\right)$, frequência de amortecimento $(\omega \mathrm{d})$, frequência natural $(\omega \mathrm{n})$, razão de amortecimento $(\xi)$, coeficiente de amortecimento $(C)$ e coeficiente de amortecimento crítico $(\mathrm{Cc})$, conforme equação (1), podemos estimar parâmetros.

$$
\omega_{d}=\frac{2 \pi}{T_{d}}=\omega_{n} \sqrt{1-\xi^{2}} ; C=2 \xi m \omega_{n} ; \xi=\frac{C}{C_{c}}=\frac{1}{n .2 \pi} \cdot \ln \left(\frac{x(t)}{x\left(t+n T_{d}\right)}\right)
$$

Objetiva-se no presente trabalho determinar valores da razão de amortecimento e coeficiente de amortecimento considerando três modelos com corpos de prova experimentais, utilizando-se o método do decremento logarítmico por meio de acelerômetro.

\section{Metodologia}

\subsection{Estrutura metodológica}

Neste item são apresentados os materiais utilizados para a entrada de dados e o sistema de aquisição, os métodos considerados para a análise dos dados coletados e os resultados observados. São apresentados também os valores da razão de amortecimento e coeficiente de amortecimento para cada modelo proposto.

\subsection{Montagem e aquisição de dados}

Para a aquisição de dados considerou-se três modelos diferentes:

1. uma régua de metal de $15 \mathrm{~cm} \quad$ (modelo 01);

2. uma régua de plástico de $30 \mathrm{~cm}$ (modelo 02); 
3. uma régua de metal de $30 \mathrm{~cm} \quad$ (modelo 03).

Inicialmente as réguas e o acelerômetro (medir aceleração ou para detectar e vibrações no sistema) foram pesados para a determinação da massa do conjunto régua/acelerômetro. Os valores obtidos são apresentados na Tabela 1.

Tabela 1 - Valores das massas dos materiais.

\begin{tabular}{cccc}
\hline Modelos & Massa das réguas $(\mathbf{g})$ & Massa do acelerômetro $(\mathbf{g})$ & Total do conjunto $(\mathbf{g})$ \\
\hline Modelo 01 & 12,760 & 7,989 & 20,749 \\
\hline Modelo 02 & 14,248 & 7,989 & 22,237 \\
\hline Modelo 03 & 34,845 & 7,989 & 42,834 \\
\hline
\end{tabular}

Fonte: Autores (2021).

O sistema utilizado para a aquisição de dados foi o Módulo da Série C de entrada de som e vibração da National Instruments TM, conectado a um notebook utilizando-se o software LabVIEW. Inicialmente, prendeu-se o acelerômetro em uma das extremidades das réguas com a outra extremidade pressa a uma morsa. Posteriormente, foi provocada a oscilação das réguas de forma manual, totalizando quatro repetições para cada modelo. A frequência de amostragem adotada foi de $500 \mathrm{~Hz}$. A Figura 1 apresenta o sistema de aquisição de dados no LabVIEW.

Figure 1 - Sistema de aquisição de dados proposto.

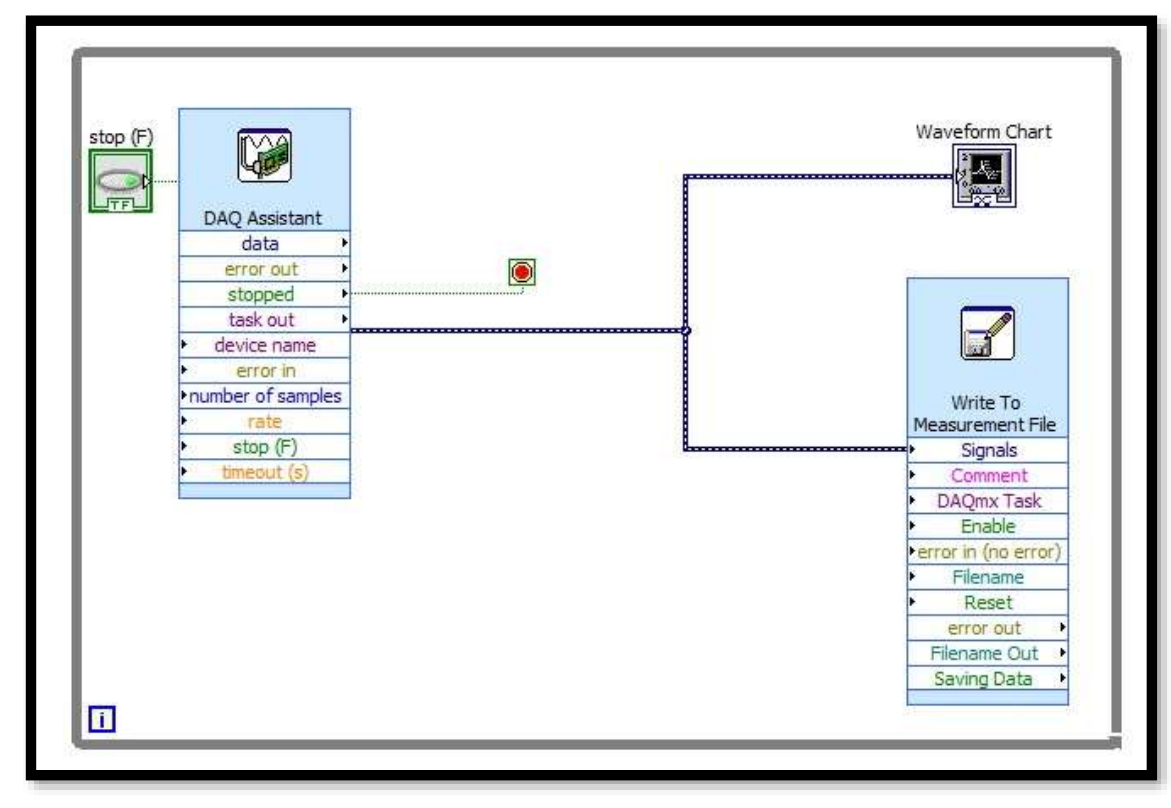

Fonte: Autores (2021).

\subsection{Análise de saída}

Após a aquisição de dados, discutida no item anterior, os mesmos foram tratados computacionalmente via software Matlab. Inicialmente levantou-se a curva amplitude por tempo para cada repetição de cada modelo. A Figura 2 apresenta a resposta para a segunda repetição do modelo 01 (régua de $15 \mathrm{~cm}$ de metal). A Figura 3 apresenta a resposta para a primeira repetição do modelo 02 (régua de $30 \mathrm{~cm}$ de plástico). A Figura 4 apresenta a resposta para a primeira repetição do modelo 03 (régua de $30 \mathrm{~cm}$ de metal). 
Figura 2 - Amplitude x Tempo em modelo 01.

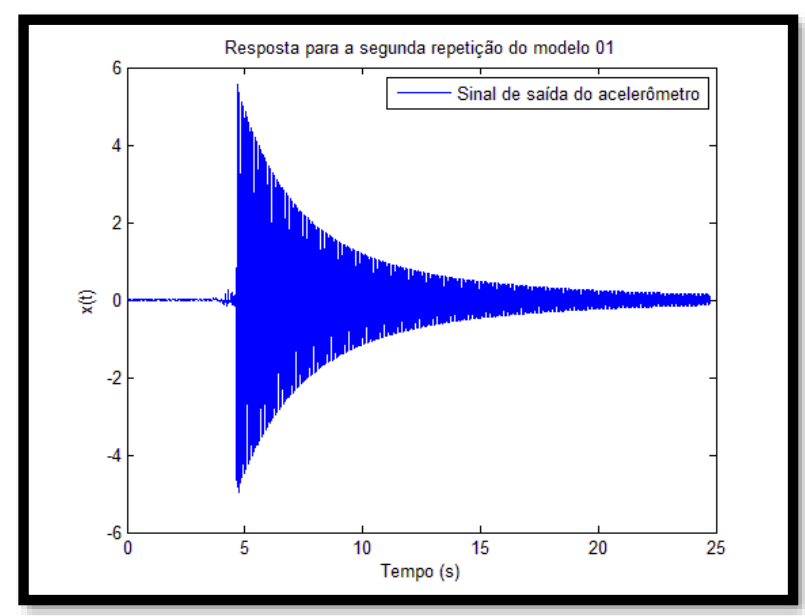

Fonte: Autores (2021).

Figura 3 - Amplitude x Tempo em modelo 02.

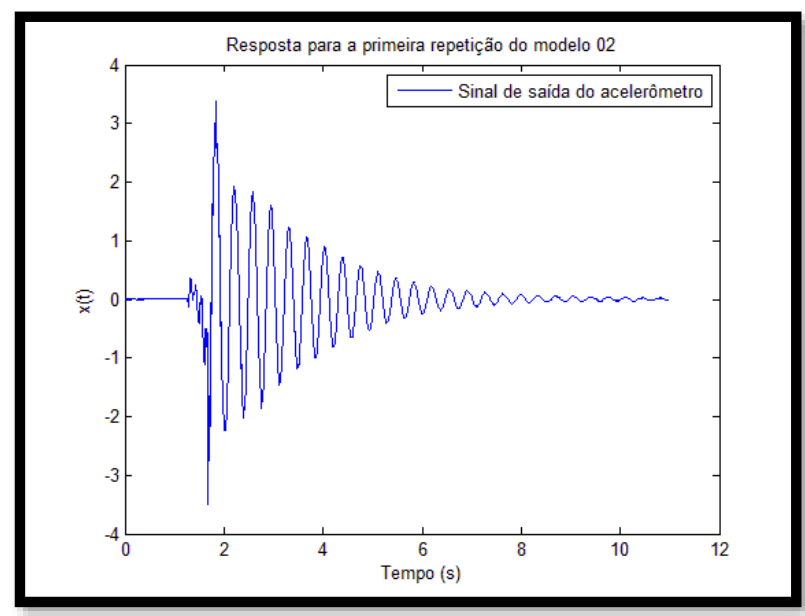

Fonte: Autores (2021).

Figura 4 - Amplitude x Tempo em modelo 03.

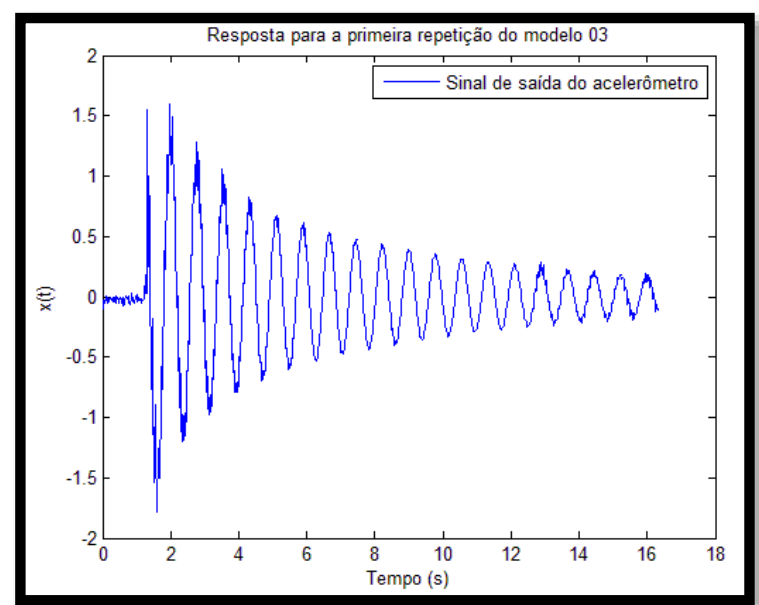

Fonte: Autores (2021). 
Posteriormente o sinal foi tratado para se calcular o tempo entre dois picos positivos, ou seja, o período do sinal $\left(\mathrm{T}_{\mathrm{d}}\right)$ e os valores de amplitude destes picos $\left(x(t)\right.$ e $\left.x\left(t+n T_{d}\right)\right)$, onde $n$ é a quantidade de ciclos considerados entre dois picos positivos. Adotou-se $n=17$ para a realização dos cálculos iniciando do primeiro ciclo. Desta forma, considera-se grande parte da curva logarítmica. Para efeito de comparação foram realizados cálculos utilizando-se $n=1$ em diferentes pontos da curva, porém os resultados não foram consistentes. A Figure 5 apresenta o sinal após o tratamento computacional, com os valores de amplitude e tempo denotados de $y$ e $x$, respectivamente.

Figure 5 - Tratamento computacional geral: Amplitude x Tempo.

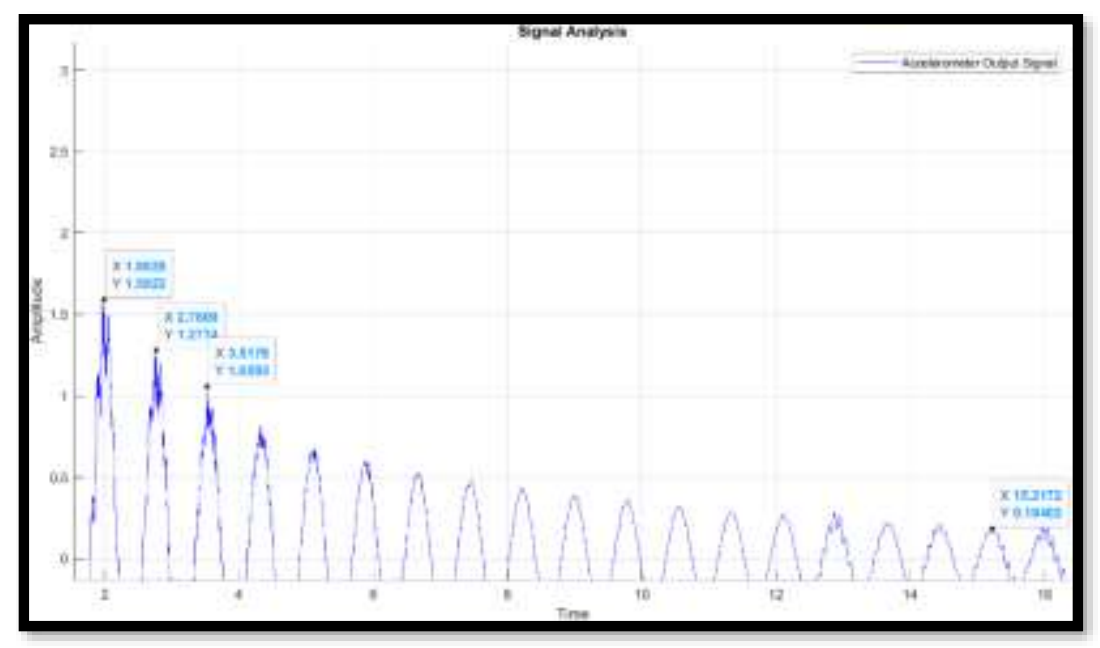

Fonte: Autores (2021).

\section{Resultados e Discussão}

A partir dos valores obtidos da Figura 4 calcularam-se os seguintes parâmetros: Td, $\omega d, \omega n, \xi$, C e Cc, conforme equação (1) citada. Os cálculos foram realizados para cada modelo considerando quatro replicações para cada. Os resultados são apresentados nas Tabelas 2, 3 e 4 .

Tabela 2 - Resultados para o Modelo 01.

\begin{tabular}{ccccccc}
\hline Replicação & $\xi$ & $\mathbf{T}_{\mathbf{d}}(\mathbf{s})$ & $\boldsymbol{\omega}_{\mathbf{d}}(\mathbf{r a d} / \mathbf{s})$ & $\boldsymbol{\omega}_{\mathbf{n}}(\mathbf{r a d} / \mathbf{s})$ & $\boldsymbol{C}\left(\frac{\boldsymbol{N}}{\boldsymbol{m}} \boldsymbol{s}\right)$ & $\mathbf{C}_{\mathbf{c}}\left(\frac{\boldsymbol{N}}{\boldsymbol{m}} \boldsymbol{s}\right)$ \\
\hline 1 & 0,0038 & 0,0824 & 76,2522 & 76,2528 & 12,1674 & 3164,30 \\
\hline 2 & 0,0047 & 0,0829 & 75,7923 & 75,7932 & 14,684 & 3145,30 \\
\hline 3 & 0,0052 & 0,0811 & 77,4745 & 77,4756 & 16,746 & 3215,10 \\
\hline 4 & 0,0052 & 0,0818 & 76,8116 & 76,8126 & 16,5663 & 3187,60 \\
\hline Média & $\mathbf{0 , 0 0 4 9 5}$ & $\mathbf{0 , 0 8 2 1}$ & $\mathbf{7 6 , 5 3 1 9}$ & $\mathbf{7 6 , 5 3 2 7}$ & $\mathbf{1 5 , 6 2 5 1 5}$ & $\mathbf{3 1 7 5 , 9 5}$ \\
\hline
\end{tabular}

Fonte: Autores (2021). 
Tabela 3 - Resultados para o Modelo 02.

\begin{tabular}{ccccccc}
\hline Replicação & $\boldsymbol{\xi}$ & $\mathbf{T}_{\mathbf{d}}(\mathbf{s})$ & $\boldsymbol{\omega}_{\mathbf{d}}(\mathbf{r a d} / \mathbf{s})$ & $\boldsymbol{\omega}_{\mathbf{n}}(\mathbf{r a d} / \mathbf{s})$ & $\boldsymbol{C}\left(\frac{\boldsymbol{N}}{\boldsymbol{m}} \boldsymbol{s}\right)$ & $\mathbf{C}_{\mathbf{c}}\left(\frac{\boldsymbol{N}}{\boldsymbol{m}} \boldsymbol{s}\right)$ \\
\hline 1 & 0,0353 & 0,393 & 15,9877 & 15,9977 & 25,1139 & 711,4825 \\
\hline 2 & 0,0356 & 0,3845 & 16,3412 & 16,3516 & 25,9213 & 727,2199 \\
\hline 3 & 0,0361 & 0,3887 & 16,1646 & 16,1751 & 25,9071 & 719,3717 \\
\hline 4 & 0,0347 & 0,3929 & 15,9918 & 16,0015 & 24,7057 & 711,6491 \\
\hline Média & $\mathbf{0 , 0 3 5 4 5}$ & $\mathbf{0 , 3 9 0 8}$ & $\mathbf{1 6 , 0 7 8 2}$ & $\mathbf{1 6 , 0 8 8 3}$ & $\mathbf{2 5 , 5 1 0 5}$ & $\mathbf{7 1 5 , 5 1 0 4}$ \\
\hline
\end{tabular}

Fonte: Autores (2021).

Tabela 4 - Resultados para o Modelo 03.

\begin{tabular}{ccccccc}
\hline Replicação & $\boldsymbol{\xi}$ & $\mathbf{T} \mathbf{d}(\mathbf{s})$ & Wd $(\mathbf{r a d} / \mathbf{s})$ & Wn $(\mathbf{r a d} / \mathbf{s})$ & $\boldsymbol{C}\left(\frac{\boldsymbol{N}}{\boldsymbol{m}} \boldsymbol{s}\right)$ & $\mathbf{C}_{\mathbf{c}}\left(\frac{\boldsymbol{N}}{\boldsymbol{m}} \boldsymbol{s}\right)$ \\
\hline 1 & 0,0202 & 0,7569 & 8,3012 & 8,3029 & 14,3701 & 711,2932 \\
\hline 2 & 0,0207 & 0,778 & 8,0761 & 8,0778 & 14,3432 & 692,0097 \\
\hline 3 & 0,0193 & 0,7593 & 8,275 & 8,2765 & 13,7145 & 709,0328 \\
\hline 4 & 0,0204 & 0,7871 & 7,9827 & 7,9844 & 13,9531 & 684,0045 \\
\hline Média & $\mathbf{0 , 0 2 0 3}$ & $\mathbf{0 , 7 6 8 6 5}$ & $\mathbf{8 , 1 7 5 5 5}$ & $\mathbf{8 , 1 7 7 1 5}$ & $\mathbf{1 4 , 1 4 8 1 5}$ & $\mathbf{7 0 0 , 5 2 1 2 5}$ \\
\hline
\end{tabular}

Fonte: Autores (2021).

Pela análise das Tabelas 2, 3 e 4, observa-se a importância da replicação do experimento, pois podem ocorrer inconsistências em dada medição. Nota-se que os valores de $\omega_{\mathrm{n}}$ e $\omega_{\mathrm{d}}$ são praticamente iguais, em conformidade com a teoria na literatura. Considera-se a média das replicações para obter um resultado mais próximo do verdadeiro fundamental.

Para o modelo 01 a partir da fórmula $\mathrm{f}=1 / \mathrm{Td}$, em que a frequência de oscilação é de $12,18 \mathrm{~Hz}, 2,56 \mathrm{~Hz}$ e $1,30 \mathrm{~Hz}$ para os modelos 01, 02 e 03 respectivamente. Para efeito de simplificação, na Tabela 5 estão os resultados para o modelo 03 considerando $n=1$ e os dois primeiros picos positivos no intuito de realizar comparação com a Tabela 4. Percebe-se uma grande diferença nos valores calculados.

Tabela 5 - Resultados para o Modelo $03 \operatorname{com} n=1$.

\begin{tabular}{ccccccc}
\hline Replicação & $\xi$ & $\mathbf{T}_{\mathbf{d}}(\mathbf{s})$ & $\boldsymbol{\omega}_{\mathbf{d}}(\mathbf{r a d} / \mathbf{s})$ & $\omega_{\mathbf{n}}(\mathbf{r a d} / \mathbf{s})$ & $\boldsymbol{C}\left(\frac{\boldsymbol{N}}{\boldsymbol{m}} \boldsymbol{s}\right)$ & $\mathbf{C}_{\mathbf{c}}\left(\frac{\boldsymbol{N}}{\boldsymbol{m}} \boldsymbol{s}\right)$ \\
\hline 1 & 0.0351 & 0.7780 & 8.0761 & 8.0810 & 24.2717 & 692.2867 \\
\hline 2 & 0.0414 & 0.7781 & 8.0750 & 8.0820 & 28.6475 & 692.3651 \\
\hline 3 & 0.0374 & 0.7047 & 8.9161 & 8.9224 & 28.5894 & 764.3605 \\
\hline 4 & 0.0338 & 0.7611 & 8.2554 & 8.2601 & 23.9489 & 707.6290 \\
\hline Média & $\mathbf{0 . 0 3 6 9}$ & $\mathbf{0 . 7 5 5 5}$ & $\mathbf{8 . 3 3 0 7}$ & $\mathbf{8 . 3 3 6 4}$ & $\mathbf{2 6 . 3 6 4 4}$ & $\mathbf{7 1 4 . 1 6 0 3}$ \\
\hline
\end{tabular}

Fonte: Autores (2021).

Hodrick e Prescott (1997) propuseram um método matemático muito relevante até os dias atuais, um filtro conhecido pelas iniciais de seus nomes (HP). No modelo 03, em primeira replicação, utilizou-se o HP Filter para eliminar o ruído presente no sinal original. O método consiste na análise estatística para tratamento de dados indexados no tempo, sinais ou séries temporais. O conceito básico é decompor o sinal em suas componentes de tendência (cíclica/aleatória). Em essência, removemse as flutuações de baixa frequência dos sinais (ou séries). A hipótese é que nessa separação a variabilidade de baixa frequência representa a tendência de prazo mais longo, e a variabilidade de frequência mais alta representa os ciclos mais curtos (e/ou aleatórios). A Figura 6 mostra a atuação do filtro no sinal do modelo 03 na primeira replicação. Percebe-se que o ruído do sinal original se apresentou na componente cíclica, proporcionando uma análise melhor do sinal na componente de tendência do $H P$ 
Filter.

Figura 6 - HP Filter aplicado ao sinal do modelo 03.

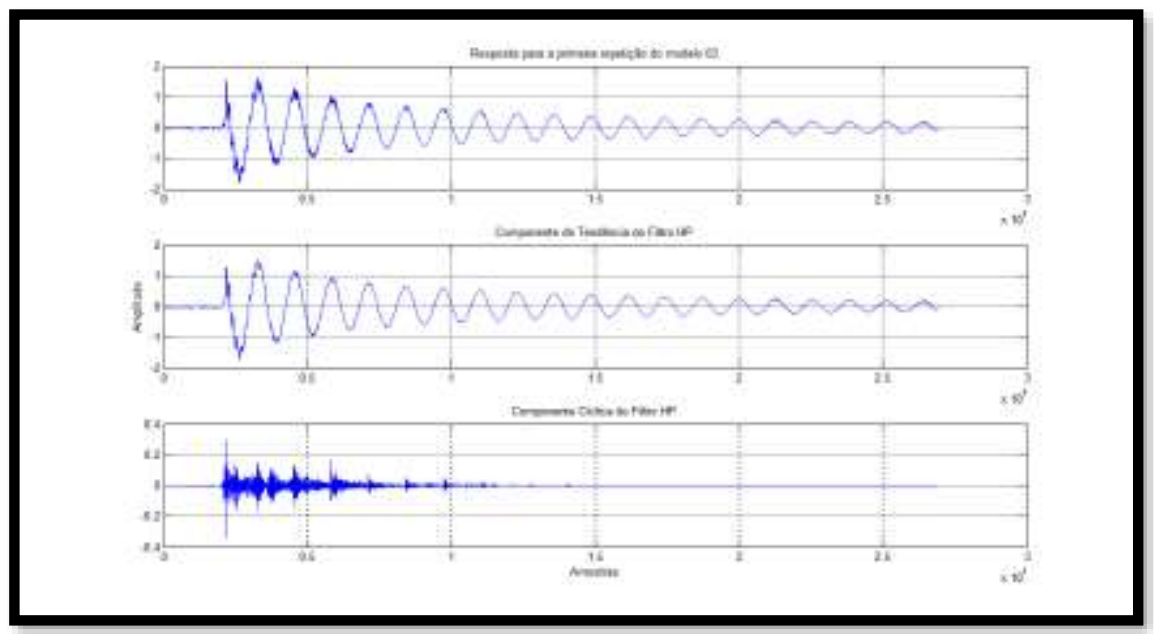

Fonte: Autores (2021).

A mesma análise na componente de tendência, conforme Figura 7, para a determinação dos parâmetros mostrados nas tabelas anteriores, porém conclui-se que o resultado não foi muito diferente, conforme atestado pela Tabela 6.

Figura 7 - Componente de Tendência.

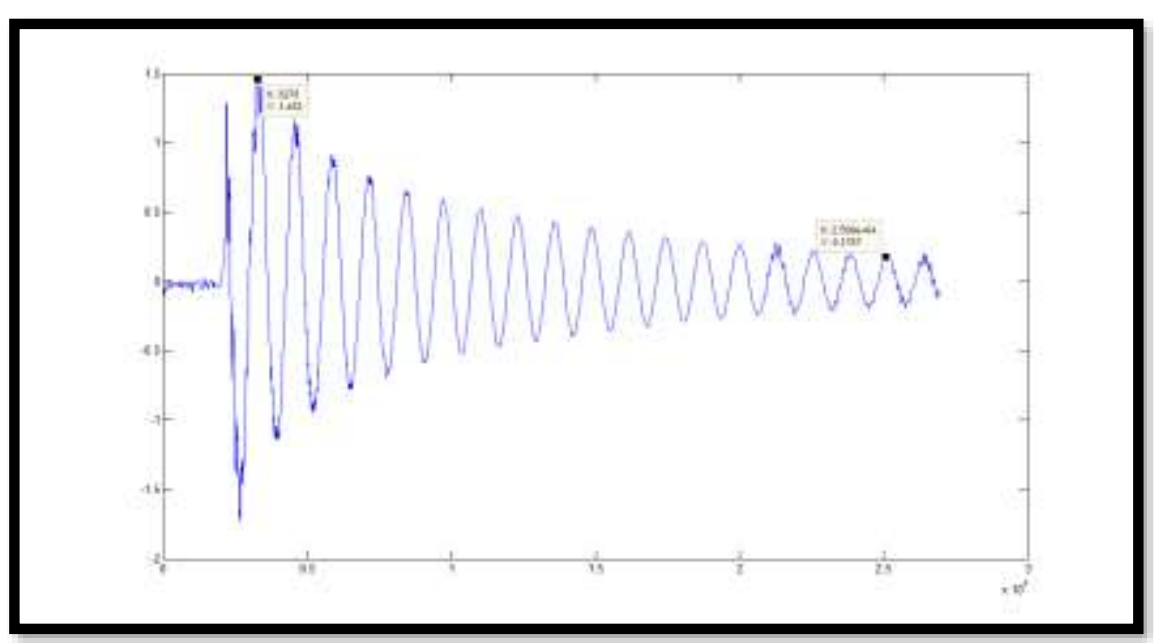

Fonte: Autores (2021).

Tabela 6 - Resultados comparativos para o Modelo 03 com $n=17$.

\begin{tabular}{ccccccc}
\hline & $\xi$ & $\mathbf{T}_{\mathbf{d}}(\mathbf{s})$ & $\boldsymbol{\omega}_{\mathrm{d}}(\mathbf{r a d} / \mathbf{s})$ & $\boldsymbol{\omega}_{\mathbf{n}}(\mathbf{r a d} / \mathbf{s})$ & $\boldsymbol{C}\left(\frac{\boldsymbol{N}}{\boldsymbol{m}} \boldsymbol{s}\right)$ & $\mathbf{C}_{\mathbf{c}}\left(\frac{\boldsymbol{N}}{\boldsymbol{m}} \boldsymbol{s}\right)$ \\
\hline Replicação 1 & 0,0202 & 0,7569 & 8,3012 & 8,3029 & 14,3701 & 711,2932 \\
\hline HP Filter & 0.0198 & 0,7569 & 8,3012 & 8,3028 & 14,0635 & 711,2870 \\
\hline
\end{tabular}

Fonte: Autores (2021). 


\section{Considerações Finais}

O amortecimento é uma das propriedades fundamentais nos estudos de tecnologias que envolvem os materiais, sendo de importância ímpar na aplicação em áreas de engenharias diversas. Para obtenção de dados precisos e com exatidão aceitável nos padrões laboratoriais, faz-se necessário o acesso a equipamentos tecnológicos avançados que permitam os ensaios em ambientes controlados. Foi possível analisar e determinar parâmetros fundamentais associados ao amortecimento utilizando o método do decremento logarítmico, e desenvolvendo assim uma metodologia para uso na literatura.

$\mathrm{O}$ trabalho pode ser ampliado para trabalhos futuros por meio de acesso à dispositivos mais robustos e com mais investimentos na padronização dos materiais utilizados e ambiente controlado. $\mathrm{O}$ uso de acelerômetros mais atuais em conjunto com os softwares abordados no trabalho, podem contribuir com análises mais precisas e exatas, bem como combinar distintas localizações de sensores e efetuar comparativos de modelos de maior otimização baseados em posicionamento. Sua aplicação pode ser expandida para diversas áreas, e contribuir para o desenvolvimento de sistemas preventivos.

\section{Agradecimentos}

Os autores agradecem a CAPES (Coordenação de Aperfeiçoamento de Pessoal de Nível Superior) pelo suporte.

\section{Referências}

Anwar, T. S., Rosmaliati, Adila, A. F., Lystianingrum, V., Priyadi, A., \& Purnomo, M. H. (2019). Design and Implementation of a piezoelectric accelerometer using Arduino Mega for detection of vibration on the $20 \mathrm{kV}$ transformer tank. 2018 International Seminar on Intelligent Technology and Its Applications (ISITIA), 413-418.

Cossolino, L. C., \& Pereira, A. H. A. (2010). Amortecimento: classificação e métodos de determinação. Universidade de São Carlos.

Figliola, R. S., \& Beasley, D. E. (2014). Theory and design for mechanical measurements. John Wiley \& Sons.

Hasibuzzaman, M., Shufian, A., Shefa, R. K., Raihan, R., Ghosh, J., \& Sarker, A. (2020). Vibration Measurement \& Analysis Using Arduino Based Accelerometer. 2020 IEEE Region 10 Symposium (TENSYMP), 508-512.

Hoang, M. L., \& Pietrosanto, A. (2021). A New Technique on Vibration Optimization of Industrial Inclinometer for MEMS Accelerometer Without Sensor Fusion. IEEE Access, 9(2021), 20295-20304.

Hodrick, R., \& Prescott, E. C. (1997). Postwar U.S. Business cycles: An empirical investigation. Journal of Money, Credit, and Banking, 29(1), 1-16.

Ismail, M. I. M., Dziyauddin, R. A., Salleh, N. A. A., Sukki, F. M., Bani, N. A., Izhar, M. A. M., \& Latiff, L. A. (2019). A Review of vibration detection methods using accelerometer sensors for water pipeline leakage. IEEE Acess, 7(2019), 51965-51981.

Kasraei, A., \& Zakeri, J. A. (2020). Effective time interval for railway track geometry inspection. Archives of Transport, 53(1), 53-65.

Keck, A., Pott, J. U., \& Sawodny, O. (2015). Accelerometer-based online reconstruction of vibrations from delayed measurements. 2015 IEEE Conference on Control Applications (CCA), 424-429.

Koene, I., Viitala, R., \& Kuosmanen, P. (2019). Internet of things based monitoring of large rotor vibration with a microelectromechanical systems accelerometer. IEEE Access, 7(2019), 92210-92219.

Lazan, B. J. (1968). Damping of materials and members in structural mechanics. Pergamon Press.

Lima, J. B., Lopes, L. A. S., \& Filho, L. A. C. M. A. (2021). Contribution to the maintenance of the permanent track with accelerometers in portable inertia packages. Research, Society and Development, 10(6), e3910615369.

Liu, J., Wang, P., \& Tian, X. (2017). Vibration displacement measurement based on three axes accelerometer. 2017 Chinese Automation Congress (CAC), 23742378 .

Mohanty, S., Gupta, K. K., Raju, K. S., Mishra, V., Kumar, V., \& Prasad, B. (2014). Characterization of wireless accelerometer sensor and its industrial applications. 2014 Twentieth National Conference on Communications (NCC), 1-5.

Nakamura, S., Higashi, Y., Masuda, A., \& Tanaka, A. (2018). Development of Vibration Measurement Unit for Steel Structure Using MEMS Accelerometer. 2018 IEEE Region 10 Conference, 0587-0592.

Pereira, A. H. A., Musolino, B., Maciel, C. D., \& Rodrigues, J. A. (2012). Algoritmo para determinação do amortecimento de materiais cerâmicos pela técnica das frequências naturais de vibração via excitação por impulse. Cerâmica, 58(346), 229-237.

Singiresu, R. (2009). Vibrações mecânicas. [S.1.]: Pearson. 
Research, Society and Development, v. 10, n. 11, e235101119438, 2021

(CC BY 4.0) | ISSN 2525-3409 | DOI: http://dx.doi.org/10.33448/rsd-v10i11.19438

Teixeira, P. S., Silva, A. J., \& Feiteira, J. F. S. (2014). Avaliação e comparação de características de amortecimento de sinais gerados de diferentes violões. Cadernos UNIFOA, 9(26), 17-30.

Thukral, V., Cahu, M., Zaal, J. J. M., Jalink, J., Roucou, R., \& Rongen, R. T. H. (2019). Assessment of Accelerometer Versus LASER for Board Level Vibration Measurements. 2019 IEEE 69th Electronic Components and Technology Conference (ECTC), 1339-1346.

Valinho, M. N., Cescon, J. T. A. M., Simoni, A. P. R., \& Zambrotti, L. de L. P. (2020). Socioeconomic impacts with the implementation of the railroad EF-118 RJ/ES: comparison of cargo logistics at the Port of Açu. Research, Society and Development, 9(9), e995998389. 